\title{
Sparse Bilateral Denoising for CT Scan Images with Edge Preservation
}

\author{
Veni N, Manjula J, Vivek Maik
}

\begin{abstract}
Denoising in CT images using bilateral with sparse representation is presented in this paper. Artifacts occurs in images when an $X$-ray penetrates the thick objects like bones, implanted organs, surgical clips etc.,. Due to these artifacts in images, the quality of artifact pruning algorithms will be diminished. In order to preserve the image quality as well as edge details, a bilateral filter along with sparse representation is proposed to reduce the noises. The proposed technique is applied to CT humorous bone image and has achieved the better PSNR of $22 \mathrm{~dB}$ approximately for $512 \times 512$ image as compared to bicubic filter. The simulated real datasets are used to quantitatively evaluate the noise. Moreover the proposed denoising approach can outperform the latest approach in terms of fidelity
\end{abstract}

Keywords: CT image, Denoising, Bilateral filter, Sparse representation, Dictionary learning.

\section{INTRODUCTION}

Image denoising is the reconstruction of the original images from a noisy image. Image denoising is one of the essential pre-process step to upgrade the potential of subsequent applications in image processing such as segmentation, restoration, enhancement, recognition, compression etc.,

Denoising is also called inverse problem, aims to reconstruct a clean image from its noisy measurements. As the image acquisition time is limited due to patient inconvenience, the quality of the image gets degraded results in blurring boundaries and loss of structural details. All together reduces the efficiency of diagnosis. Filtering the images with complex structures by fixed non-adaptation filter results in blurring.

Mostly real images will have some levels of noise or blur distortions which will degrade the image. In general these degradations is denoted as in equation (1),

$y=a x+n$

In the above equation, y represents the measured image $\mathrm{x}$ represents the input image and $\mathrm{n}$ denotes noise in image. In $\mathrm{CT}$, the reconstructed image may be noisy due to nonlinear attenuation characteristics of X-ray. The amount of noise depends on the measuring devices used for scanning. The measuring devices introduces more artifacts in the images of dense objects. There are various approaches to study and reduce these artifacts.

\footnotetext{
Revised Manuscript Received on August 14, 2019.

Veni N, Department of ECE, SRMIST, Chennai, Tamilnadu, India (email: veni.n@ktr.srmuniv.ac.in)

Mnjula J, Department of ECE, SRMIST, Chennai, Tamilnadu, India (email: manjula.j@ktr.srmuniv.ac.in)

Vivek Maik, Department of ECE, SRMIST, Chennai, Tamilnadu, India (email: vivekmaik.d@ktr.srmuniv.ac.in)
}

In almost all medical imaging approaches, it is required to smoothen the image while preserving details. However linear filters such as mean and gaussian filters[4] will introduce blurring while removing noise. The blurring intensity is corresponding to the degree of neighbourhood regions. Whereas nonlinear filter generally maintain edge details while noise removal. A conventional median filter is a famous nonlinear spatial filter, in which the intensity of each pixel is replaced with centre value from neighbouring pixels.

In general Image denoising can be done in two ways: model based methods and learning-based methods. Image denoising is done by using some image and noise prior models in model based methods, where as in learning based methods, a noisy image is transformed to noise-free image by mapping function based on learning. In this paper our approach is dictionary learning based approach.

In this paper a traditional non-linear edge-preserving bilateral filtering combined with sparse representation is used. Since the denoised image obtained by sparse based method suppress the more details, this paper uses bilateral filter along with sparse to preserve those details. The technique proposed in this paper not only reduce the noise but also retains the structural information.

Organization of the paper : The rest of this paper is organised as follows: Section II discuss the related works carried out in image denoising, in section III sparse representation is addressed and the experimental results are demonstrated in section IV.

\section{RELATED WORKS}

A conventional bilateral is a famous nonlinear spatial filter, which replaces the intensity of each and every pixel (1) with weighted average of nearby pixels. This weight varies in accordance with gaussian distribution. The filter kernel of bilateral filter are : spatial filter kernel and a range filter kernel, which uses all the pixels which are closer and similar to centre pixels, hence extreme edges are preserved.

A bilateral filter was developed from a Bayesian approach with the help of penalty function also obtained a bilateral filter by single iteration of diagonal normalized steepest descent approach and extended the bilateral filter to reconstruction problem[8],separable bilinear filter which filters the image in one dimension and there upon filtered in another dimension and it is a good approximation of kernel filtering, extremely fast but effective filtering kernel is

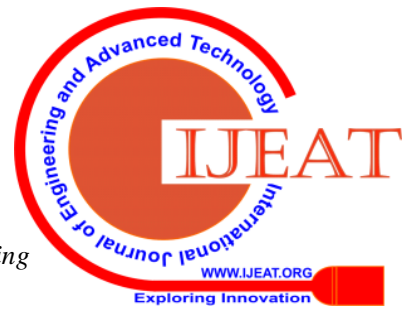


distorted [9].A fast bilateral filter discussed in [10] is to improve the accuracy by down sampling the intermediate images. Image denoised by sparse method suppress more details [6].The modern filtering and representative methods use image adaptive processing, such filters are bilateral filter[1], non-local means[2] filters. In state of the art image denoising approaches edge blurring is still inevitable and artifacts in smooth regions of images. Moreover the bicubic filter uses 16 pixels ie. $4 \times 4$ and the filtered image obtained by bicubic filter are smoother but some artefacts are present in it, at the same time the bilateral filter uses only 4 pixels hence preserve more edge details.

Several algorithms have been presented, to find the sparset representation such as matching pursuit [13] ,in which only single atom is obtained in each step, and determined the next atom by using the previous one. Orthogonal matching pursuit in which after each step the coefficients are recapped [14], stagewise orthogonal matching pursuit [15] and subspace pursuit [16] and in Basis pursuit, they used L1 norm as penalty. The existing algorithms seeking for an appropriate dictionary [17], [18].The overcomplete dictionary is learned from image patches and is applied to denoising problem using K-SVD [19].

Sparse coding approaches used to update the coefficients while keeping D fixed. Analysis SimCo algorithms [11] proposed to update the several atoms simultaneously in every iteration with lesser penalty terms and eliminated the similar atoms appeared in the learned dictionary. Simultaneous Low-Pass Filtering and Total Variation denoising [12] in which a low pass signal and a sparse signal was determined by concurrent use of low pass filtering and sparsity denoising.

In this paper a traditional non-linear edge-preserving bilateral filtering combined with sparse representation is used. Since the denoised image obtained by sparse based method suppress the more details, this paper uses bilateral filter along with sparse to preserve those details. The technique proposed in this paper not only reduce the noise but also retains the structural information.

\section{SPARSE REPRESENTATION}

The goal of image denoising is to retrieve a high quality image $\mathrm{x}$ from a deteriorate version of it, $\mathrm{y}$. As similar to expression (1) replacing $\mathrm{n}$ with white gaussian noise $\mathrm{w}$ with zero mean and standard deviation $\sigma$, the equation is rewritten as in equation (2),

$$
y=a x+w
$$

$\mathrm{x}, \mathrm{y}, \mathrm{w} \in \mathrm{Rm}^{*} \mathrm{n}$ where $\mathrm{m}, \mathrm{n}$ are the number of pixels in the image. The clean image can be estimated from the noisy image, obtaining nearer to original image. The fundamental principle of the denoising method established in [4] and [5] represents every pixel as a linear combination of a little portion of patches captured from a fixed dictionary. This will describe the denoising function as an energy reduction procedure. The key point of dictionary learning is giving the sparse representation of the training set in order to keep the error as low as possible.

Sparse representation is the representation of as much information as possible with as less resources as possible. It is obtained by sparse dictionary learning method. In this model a signal, $\mathrm{x} \in \mathrm{R}^{\mathrm{m}} \mathrm{Rm}$, is represented as a linear combination of some atoms of dictionary $\mathrm{D} \in \mathrm{R}^{\mathrm{m} * \mathrm{n}}$. Mostly the dictionary is assumed to be overcomplete with $n>m$. This property leads to redundancy in atom but provide an improvement in sparsity. The elements of dictionary is called atoms .The sparsity of the signal $y$ will be reflected when the no. of atoms used to represent $\mathrm{x}$ is lesser than the total no. of atoms in dictionary.

Mathematically the sparse model is described as in equation (3),

$x=D \alpha$

where $\alpha$-is the coefficient vector . The nonzero elements of $\alpha$ helps in synthesising $\mathrm{x}$ through their linear combination. Two main challenges associated with this model are: i. Sparse coding problem and ii. finding a suitable dictionary to give the best set of representation as sparsely as possible for a given set of training examples.

In general most of the proposed approaches aims at minimizing of energy function, is given by equation (4),

$$
f(x)=\frac{1}{2}|| X-Y||_{2}^{2}+\operatorname{Pr}(X)
$$

In equation (4) the first term is called the optimization and the second term is called the prior. For sparse an redundant, the second term is defined by equation (5)

$$
\operatorname{Pr}(X)=\lambda|| \alpha||_{0}^{0}
$$

In our proposed methodology the coefficient vector $\alpha$ is determined by keeping the dictionary $\mathrm{D}$ fixed for a given $\mathrm{x}$.

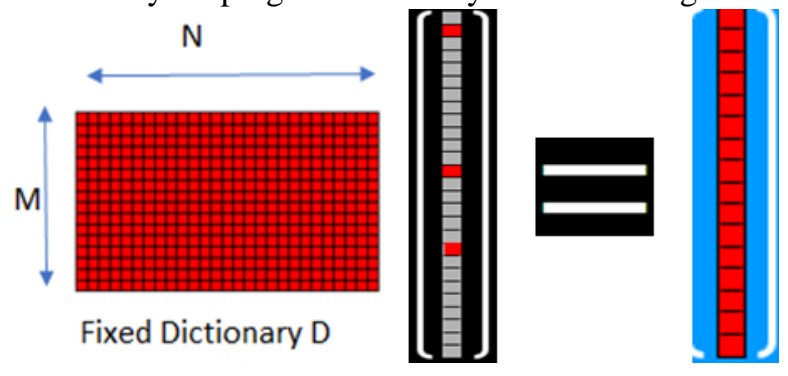

Figure 1: Dictionary Learning for $\alpha$

Figure 1 indicates the dictionary learning $\mathrm{D}$ for $\alpha$ and from which the value of $x$ can be obtained using $x=D \alpha$. The sparse representation is calculated for a fixed dictionary then the obtained sparse vector is used to fit the best representation of each image from the image patches in D. This gives the sharper edges and clear textures. $\mathrm{x}=\mathrm{D} \alpha$ is under-determined linear system for the overcomplete dictionary when $\alpha$ is not known. Even though it has number of possible solutions, the sparsity is the desired one. It can be measured using the L0 norm, which is achieved by $\min \|\alpha\|_{0}^{0}$ such that $\mathrm{x}=\mathrm{D} \alpha$. The sparse constraint optimization is given by an equation (6)

$$
\hat{\alpha}=\underset{\alpha}{\operatorname{argmin}}|| \alpha||_{0} \text { s.t. }|| D \alpha-\left.y\right|_{2} ^{2}
$$

It can be incorporated in the denoising problem statement $\mathrm{y}=\mathrm{Hx} \quad$ s.t. $\mathrm{x}=\mathrm{D} \alpha$, as in equation (7)

$$
\hat{\alpha}=\underset{\alpha}{\operatorname{argmin}}|| \alpha||_{0} \text { s.t. }|| H D \alpha-y \|\left.\right|_{2} ^{2}
$$


This can be solved more efficiently by converting to unconstrained optimization as in equation (8),

$$
\hat{\alpha}=\operatorname{argmin}|| \alpha||_{*}+\lambda|| H D \alpha-y||^{2}+B_{x}|| H D \alpha-y\left\|^{2}+B_{y}|| H D \alpha-y\right\|^{2}
$$

The equation (8) introduces three terms to remove the constraints in earlier methods.

(a) $\lambda$ is lagrangian penalty function which converges the solution towards convex minimum by giving greater penalty to the diverging solutions.

(b) $\mathrm{Bx}, \mathrm{By}$ are the bilateral filter coefficients in horizontal and vertical directions.

$\mathrm{Bx}$ and By are given by an equation (9) and (10)

$$
\begin{aligned}
& B_{x}=\frac{1}{w_{p}} \sum_{x_{i} \in \Omega} I_{\left(x_{i}\right)} f\left(\left|I_{\left(x_{i}\right)}-I_{x}\right| \mid\right) g_{s}\left(|| x_{i}-x||\right) \\
& B_{y}=\frac{1}{w_{p}} \sum_{y_{i} \in \Omega} I_{\left(y_{i}\right)} f\left(|| I_{\left(y_{i}\right)}-I_{y}||\right) g_{s}\left(|| y_{i}-y \mid\right)
\end{aligned}
$$

The use of the bilateral filter helps to remove noise and keep edge details .

$$
\hat{\alpha}=\min _{\alpha}|| H D \alpha-y||^{2}+\lambda|| H D \alpha||_{1}+B_{x y}|| y \|_{2}
$$

The equation (11) is much more efficient as the lagrangian penalty is used for $\mathrm{HD} \alpha$ and bilateral applied to y.

$$
\hat{\alpha}_{k+1} \in \lambda_{k+1}+B_{x y}(k+1)
$$

With each iteration the variable $\lambda$ and degree of filtering is varied in equation (12).

$$
\min _{H}|| H x-y\left\|^{2}+\lambda|| \nabla y\right\|^{2}
$$

After $\hat{\alpha}$ is minimized we still need to minimize for $\mathrm{H}$ to ensure full denoising in equation (13). The whole algorithm is given below.

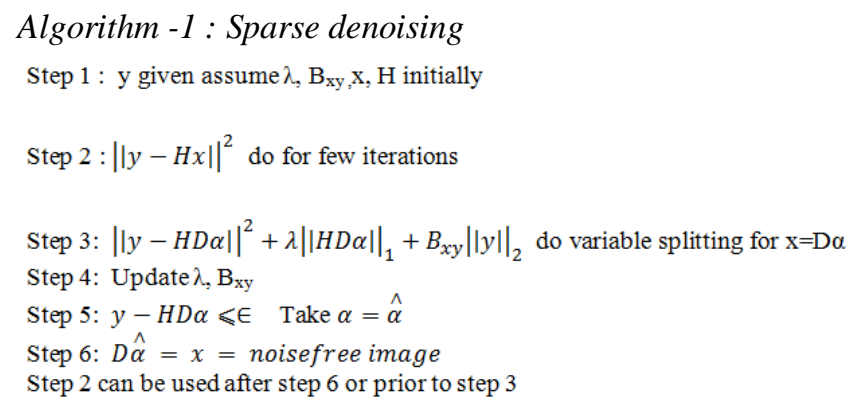

\section{EXPERIMENTAL RESULTS}

The experiments were carried out on humorous CT images which was taken from https://isbweb.org/data/vsj. The image datasets used in this experiment were from the laboratory of Human Anatomy and Embryology, University of Brussels (ULB) ,Belgium. The number of slices in the image are 102 , the number of pixels in each slice is 512 $x 512$. The first ten slices of the image were used for the denoising experiments. The proposed sparse bilateral method was applied to each slice individually. The performance is determined by calculating the PSNR of each slice.

The simulation results shown in figure 2 is obtained by applying the proposed algorithm on first ten slices of CT Humorous bone dataset. For the noisy input image of each slice, the corresponding sparse bilateral image is shown below that.
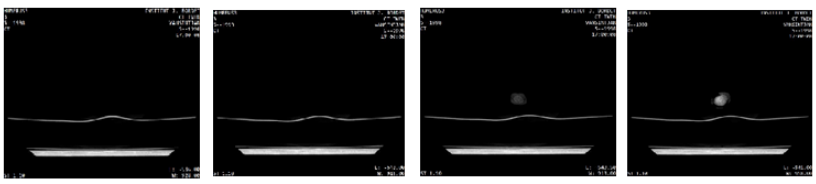

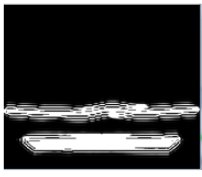

Slice 1
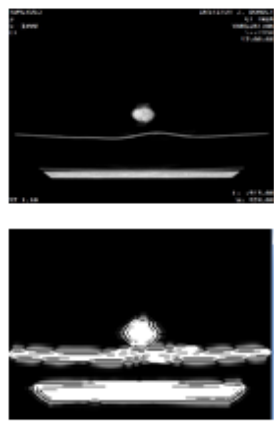

Slice 5
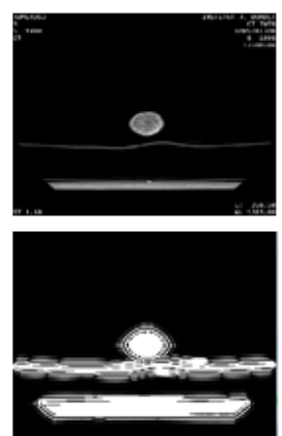

Slice 8

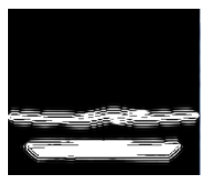

Slice 2

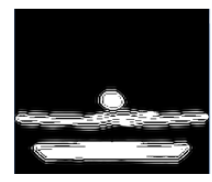

Slice 3

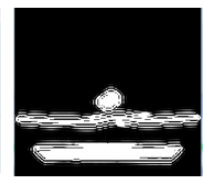

Slice 4
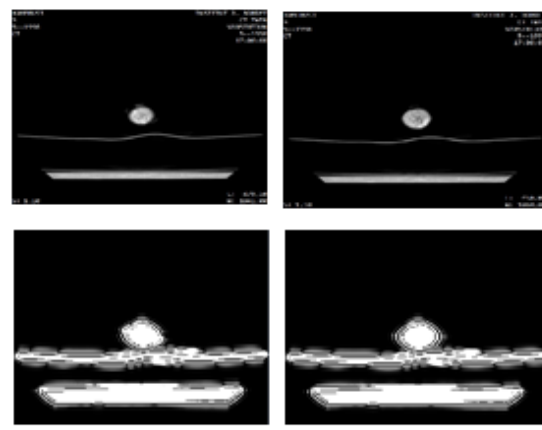

Slice 6

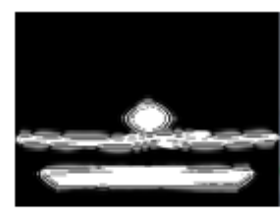

Slice 7
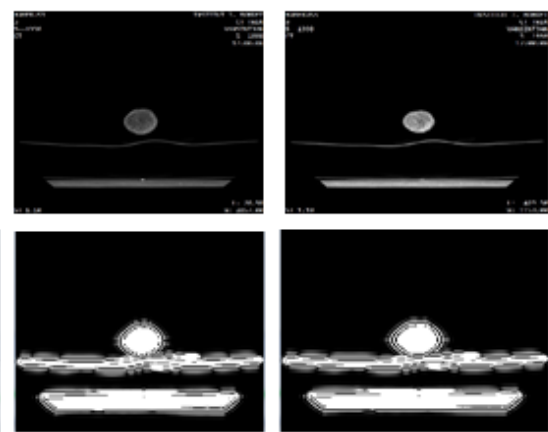

Slice 9

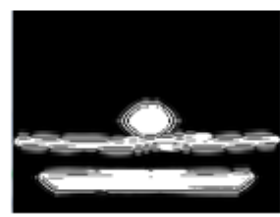

Slice 10

Figure 2 : CT image dataset with noisy image and the corresponding sparse bilateral image of slice 1 to 10

Table I illustrates the performance comparison of PSNR calculation of first ten slice of CT humorous bone images.

Table I -PSNR Comparison in $\mathrm{dB}$

\begin{tabular}{|c|c|c|}
\hline $\begin{array}{l}\text { CT Humorous } \\
\text { Bone Dataset } \\
\text { Image }\end{array}$ & $\begin{array}{l}\text { Bicubic } \\
\text { Interpolation }\end{array}$ & $\begin{array}{l}\text { Proposed Sparse } \\
\text { Bilateral } \\
\text { Representation }\end{array}$ \\
\hline Slice 1 & 21.16 & 22.29 \\
\hline Slice 2 & 21.19 & 22.31 \\
\hline Slice 3 & 21.23 & 22.39 \\
\hline Slice 4 & 21.88 & 22.39 \\
\hline Slice 5 & 21.13 & 22.25 \\
\hline Slice 6 & 21.08 & 22.22 \\
\hline Slice 7 & 20.98 & 22.12 \\
\hline Slice 8 & 20.86 & 21.94 \\
\hline Slice 9 & 20.74 & 21.85 \\
\hline Slice 10 & 20.63 & 21.71 \\
\hline
\end{tabular}

On comparison it shows that the bilateral sparse representation method has better PSNR value of approximately $6 \%$ of increase as compared to bicubic, hence it is most suitable for denoising approach for CT images.

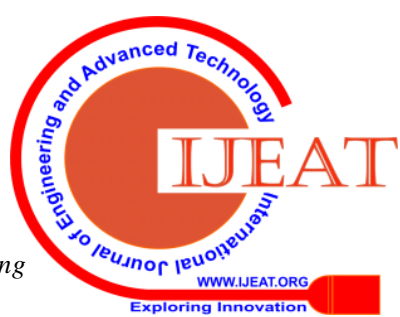


The proposed algorithm on a humorous CT images shows that the boundaries and structural details are well preserved. The obtained results indicates that the proposed method achieves the better peak signal to noise ratio than bicubic filtering, so the sparse bilateral outperforms in image denoising .The experimental indicates that the algorithm is effective for low -illumination and noise reduction.

\section{CONCLUSION}

In this paper, an image denoising algorithm using bilinear sparse representation was presented. Here the number of training iteration varies with noise level. There is an improvement in denoising rarely when the number of iterations are more for higher noise level. The overall noise performance of the method depends on various parameters of the proposed algorithm which is tuned after series tests have been performed. The denoising method proposed in this paper is analysed with ten distinct images with various noise level and achieved the better PSNR value of $22 \mathrm{~dB}$ approximately for $512 \times 512$ image as compared to bicubic filter.

\section{REFERENCES}

1. Tomasi and R. Manduchi, "Bilateral Filtering for Gray and Color Images", Proceedings of the 1998 IEEE International Conference on Computer Vision , Bombay, India, 1998

2. A. Buades, B. Coll, and J.M. Morel, "A review of image denoising algorithms, with a new one," Multiscale Modelling and Simulation, vol. 4, no. 2, pp. 490-530, 2005.

3. Matan Protter and Michael Elad "Image Sequence Denoising via Sparse and Redundant Representations", IEEE Trans. Image Process, vol. 18, NO. 1, 2009.

4. Michael Elad Michal Aharon "“ Image Denoising Via Learned Dictionaries and Sparse representation" ,Proceedings of the 2006 IEEE Computer Society Conference on Computer Vision and Pattern Recognition 2006.

5. Michael Elad and Michal Aharon, ,'Image Denoising Via Sparse and Redundant Representations Over Learned Dictionaries", IEEE Trans. Image Process, vol 15,no. 12,2006.

6. Lu Zhang,Jiaming chen Yuemin zhu, Jianhua Luo",Comparison of several new denoising methods for medical images", 3rd International Conference on Bioinformatics and Biomedical Engineering,2009.

7. Qiao Yang, Andreas Maier, Nicole Maass, Joachim Hornegger ,"Edge-Preserving Bilateral Filtering for Images Containing Dense Objects in CT", IEEE Nuclear Science Symposium and Medical Imaging Conference (2013 NSS/MIC),2013, DOI: 10.1109/NSSMIC.2013.6829358.

8. M. Elad, "On the origin of the bilateral filter and ways to improve it", IEEE Trans. Image Processing, vol.11,no.10,2002.

9. T. Q. Pham, L. J. van Vliet. ,"Separable bilateral filtering for fast video preprocessing",. IEEE International Conference on Multimedia and Expo, 2005,DOI: 10.1109/ICME.2005.1521458.

10. Paris, F. Durand.,"A fast approximation of the bilateral filter using a signal processing approach”,. International Journal of Computer Vision, 2009, 81(1):24-52.

11. Jing Dong, Wenwu Wang, Wei Dai, Mark D. Plumbley, Zi-Fa Han, and Jonathon Chambers, "Analysis SimCO Algorithms for Sparse Analysis Model Based Dictionary
Learning”, IEEE Trans. Signal Process, vol.64, no.2, 2016 .

12. Ivan W. Selesnick, , Harry L. Graber, Douglas S. Pfeil, and Randall L Barbour , "Simultaneous Low-Pass Filtering and Total Variation Denoising", IEEE Trans. Signal Process, vol .62, no.5, 2014.

13. S.G. Mallat and Z. Zhang, "Matching pursuits with timefrequency dictionaries", IEEE Trans. Signal Process. vol. 41, no. 12, pp. 3397-3415, 1993.

14. Y.C.Pati, R.Rezaiifar, and P. Krishna prasad, "Orthogonal matching pursuit: Recursive function approximation with applications to wavelet decomposition", in Proc.27th Asilomar Conference on Signals, Systems and Computers., 1993, pp. 40-44.

15. D. L. Donoho, Y. Tsaig, I. Drori, and J.-L. Starck, "Sparse solution of underdetermined systems of linear equations by stagewise orthogonal matching pursuit", IEEE Trans. Inf. Theory, vol. 58, no. 2, pp. 1094-1121, 2012.

16. W.Daiand,O.Milenkovic, "Subspace pursuit for compressive sensing signal reconstruction", IEEE Trans. Inf. Theory, vol. 55, no. 5, pp. 2230-2249, 2009.

17. K.Engan,S.Aase, and J.Hakon-Husoy , "Method of optimal directions for frame design", in Proc.Int.Conf. Acoust., Speech, Signal Process., 1999, vol. 5, pp. 2443-2446.

18. W.Dai, T.Xu, and W.Wang, "Simultaneous codeword optimization (SimCO) for dictionary update and learning", IEEE Trans. Signal Process., vol. 60, no. 12, pp. 6340-6353, 2012.

19. M. Aharon, M. Elad, and A. Bruckstein, "K-SVD: An algorithm for designing overcomplete dictionaries for sparse representation", IEEE Trans.SignalProcess., vol.54,no.11,pp.4311-4322, 2006. 